\title{
Local contact potential difference of molecular self-assemblies investigated by Kelvin probe force microscopy
}

\author{
Evan J Spadafora, Mathieu Linares, Wan Zaireen Nisa Yahya, Frederic Lincker, \\ Renaud Demadrille and Benjamin Grevin
}

\section{Linköping University Post Print}

N.B.: When citing this work, cite the original article.

Original Publication:

Evan J Spadafora, Mathieu Linares, Wan Zaireen Nisa Yahya, Frederic Lincker, Renaud Demadrille and Benjamin Grevin, Local contact potential difference of molecular selfassemblies investigated by Kelvin probe force microscopy, 2011, Applied Physics Letters, (99), 23, 233102.

http://dx.doi.org/10.1063/1.3662850

Copyright: American Institute of Physics (AIP) http://www.aip.org/

Postprint available at: Linköping University Electronic Press http://urn.kb.se/resolve?urn=urn:nbn:se:liu:diva-75291 


\title{
Local contact potential difference of molecular self-assemblies investigated by Kelvin probe force microscopy
}

\author{
Evan J. Spadafora, ${ }^{1}$ Mathieu Linares, ${ }^{2}$ Wan Zaireen Nisa Yahya, ${ }^{1}$ Frédéric Lincker, ${ }^{1}$ \\ Renaud Demadrille, ${ }^{1}$ and Benjamin Grevin ${ }^{1, a)}$ \\ ${ }^{1}$ CEA-INAC-UMR5819 SPrAM (CEA-CNRS-UJF), 17 rue des Martyrs, 38054 Grenoble Cedex 9, France \\ ${ }^{2}$ Department of Computational Physics, IFM, Linköping University, S-58183 Linköping, Sweden
}

(Received 5 August 2011; accepted 19 October 2011; published online 5 December 2011)

\begin{abstract}
Self-assembled pi-conjugated oligomer nanowires have been investigated by frequency modulation atomic force microscopy and amplitude modulation Kelvin probe force microscopy under ultra high vacuum. The distance dependence of the contact potential difference (CPD) has been analyzed by combining high resolution imaging with distance-spectroscopy measurements. It is shown that the apparition of a damping contrast characterizes the onset of short range electrostatic (SRE) forces, which are responsible for the occurrence of local CPD (LCPD) modulations correlated with the molecular lattice. By working at the onset of the damping contrast, the tip-surface separation can be adjusted to minimize the contribution of SRE forces to the measured CPD. (C) 2011 American Institute of Physics. [doi:10.1063/1.3662850]
\end{abstract}

This past decade, Kelvin probe force microscopy (KPFM) has been intensively used for near field investigations of the work function of a wide variety of materials and for local mapping of the surface potential of photovoltaic thin films ${ }^{1,2}$ and organic electronic devices. ${ }^{3,4}$ Moreover several groups have reported KPFM images displaying variations of the local contact potential difference (LCPD) at atomic scale $e^{5-9}$ on semiconducting and insulating surfaces under ultra-high vacuum (UHV). Significant progress has been made towards a quantitative interpretation of these LCPD atomic contrasts, by combining state of the art experiments with theoretical investigations of the short range electrostatic (SRE) forces involved ${ }^{9,10}$ in the KPFM imaging process. In comparison, LCPD contrasts have been poorly investigated in the case of organic and molecular materials. The influence of molecular dipoles on the KPFM signal has especially been discussed in the case of porphyrin-based molecules deposited on highly oriented pyrolytic graphite ${ }^{11}$ (HOPG) and on $\operatorname{KBr}(100)$ substrates. ${ }^{12}$ Molecular CPD contrasts have also been recently reported ${ }^{13}$ for copperphthalocyanine $(\mathrm{CuPc})$ monolayers on $\mathrm{Au}(111)$, suggesting that molecular electric dipoles may be imaged by KPFM.

In this report, we investigate the nature of the LCPD contrast on molecular pi-conjugated self-assemblies, by combining topographic, damping, and CPD imaging with damping and CPD-distance spectroscopy. It is shown that a strong contribution of SRE forces is required to achieve potential contrasts correlated with the molecular lattice, which suggests the existence of proximity effects at the tipmolecule interface.

The following results have been obtained on two semiconducting molecules containing oligothiophene segments and a fluorenone central unit, labeled QTF8 (Ref. 14) (Fig. 1(a)) and FG1 (Ref. 15) (Fig. 1(d)), which synthesis has been described elsewhere. ${ }^{14,15}$ When deposited from solutions on HOPG, both molecules self-assemble into edge-on

\footnotetext{
${ }^{\text {a) }}$ Author to whom correspondence should be addressed. Electronic mail: benjamin.grevin@cea.fr.
}

pi-conjugated nanowires stacks, which structural organization has been established by combining the results of NC-AFM with molecular mechanics (MM) and molecular dynamics (MD) simulations (see our former report ${ }^{16}$ for QTF8, and the supplementary information ${ }^{17}$ for FG1). After in-situ annealing, QTF8 stacks (Figs. 1(b) and 1(c)) consist in non-polar layers ${ }^{16}$ in which the orientation of the fluorenone unit (carrying the main molecular electric dipole) alternates from one wire $(\mathrm{C}=\mathrm{O}$ bond pointing upward $)$ to the next $(\mathrm{C}=\mathrm{O}$ bond pointing downward), leading to a doubling of the lattice periodicity (ca. $7 \mathrm{~nm}$ ) with respect to the single nanowire width. ${ }^{16}$ On the contrary, FG1 assemblies (Figs. $1(\mathrm{e})$ and 1(f)) are formed by identical molecular wires (all $\mathrm{C}=\mathrm{O}$ bonds pointing upward), ${ }^{17}$ the lattice periodicity (ca. $4.5 \mathrm{~nm}$ ) being slightly larger than the width of a single molecule due to a partial adsorption of the alkyl side groups on the graphite.

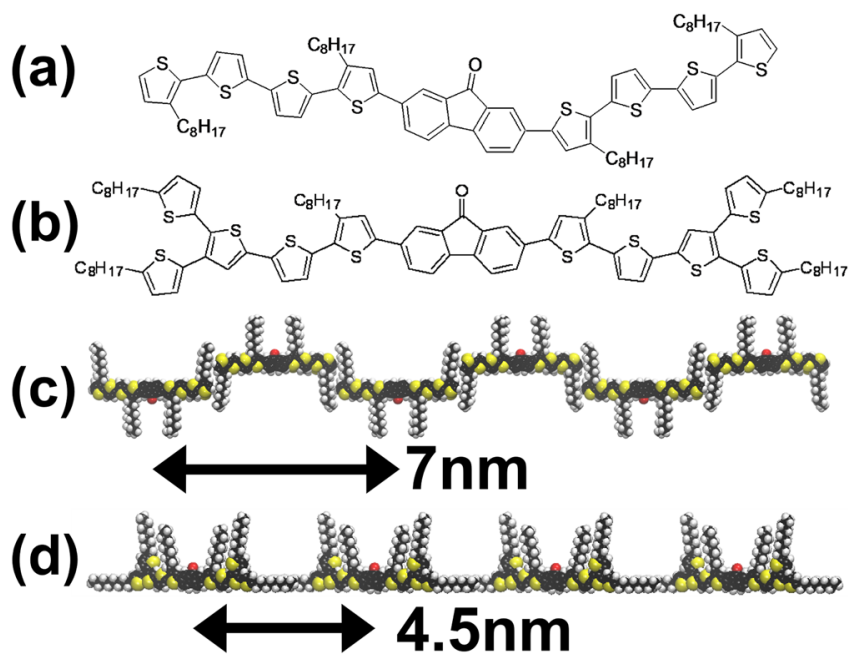

FIG. 1. (Color online) (a,b) Chemical structures of QTF8 (a) and FG1 (b). (c,d) Molecular mechanics simulations of QTF8 (c) and FG1 (d) edge-on stacks (side views). The lattice periodicity is highlighted for both molecular assemblies. Note that the plane of the conjugated systems is perpendicular to the substrate. For more details (including top views of the nanowires stacks) see Refs. 16 and 17. 
NC-AFM/KPFM experiments were carried out at room temperature in UHV using a VT-beam AFM system ${ }^{2,16}$ and in situ annealed PtIr 5 coated Si cantilevers (Nanoworld, typical spring constant $k$ of $2.8 \mathrm{Nm}^{-1}$ ). Topographic imaging was performed in the frequency modulation mode (FMAFM), and KPFM data were simultaneously recorded in the amplitude modulation mode (AM-KPFM) with a bias modulation of $100 \mathrm{mV}$ peak-to-peak (see our previous report for more details ${ }^{2}$ ). Last, distance-spectroscopy measurements were performed by recording damping $(\mathrm{z})$ and $\mathrm{CPD}(\mathrm{z})$ curves at selected locations, the feedback loop being disabled during the acquisition.

To investigate the influence of the tip-surface separation on the KPFM signal, a first series of images (topography, damping, and CPD) have been acquired at the mesoscopic scale on a FG1 thin film with sub-monolayer coverage while varying the frequency shift set point $(\Delta \mathrm{f})$ and keeping the vibration amplitude $\left(\mathrm{A}_{\mathrm{vib}}\right)$ constant (Figs. 2(a)-2(c)). The frequency shift dependence of the difference between the CPD levels (average values) measured over the oligomer stack and the substrate $\left(\triangle \mathrm{CPD}=\mathrm{CPD}_{\mathrm{FG} 1}-\mathrm{CPD}_{\mathrm{HOPG}}\right)$ has been plotted in Fig. 2(d). $\triangle$ CPD dramatically increases when lowering the set point (i.e., decreasing the tip-surface separation) below the value corresponding to the apparition of a contrast in the damping images $\left(\Delta \mathrm{f}_{\text {onset }} \approx-5 \mathrm{~Hz}\right)$, and a saturation plateau is reached below $\Delta \mathrm{f}_{\text {sat }} \approx-10 \mathrm{~Hz}$.

In the large amplitude regime, the tip-surface distance $d$ (at the lowest point of the cantilever oscillation cycle) is directly related to the normalized frequency shift, $\gamma=(\Delta \mathrm{f} \times k$ $\left.\times \mathrm{A}_{\mathrm{vib}}{ }^{3 / 2}\right) / \mathrm{f}_{0}$. A second series of image (not shown) has been recorded at variable amplitudes, but keeping a constant $\gamma=-3.7 \mathrm{fN} \mathrm{m}^{1 / 2}$ by proper adjustments of the frequency shift. In that case, the CPD is nearly unchanged whatever the amplitude set point (see the inset in Fig. 2(d)), which confirms that the tip-surface separation is the key parameter in the abovementioned frequency shift dependence of the CPD contrast.

It is also interesting to compare the measured CPD difference between the stack and the substrate, with the surface potential modification $\mathrm{DV}_{\mathrm{S}}$ expected by considering the array of the molecular dipoles oriented perpendicular to the surface. ${ }^{11,12}$ The DCPD value in the "saturation" regime corresponds to a dipole of $c a$. 2.7Debye per molecule (see the supplementary information), which is remarkably close to the values previously reported for fluorenones. ${ }^{18}$

Next, to check the occurrence of local CPD contrasts, another set of high magnification images (Fig. 3) has been recorded on the FG1 stack. First, we underline that topographic contrasts at the molecular wires scale are only achieved for tip-sample separations equal or less than the one corresponding to the onset of a contrast in the damping images $\left(\Delta \mathrm{f} \leq \Delta \mathrm{f}_{\text {onset }}\right)$. For greater tip-surface distances $\left(\Delta \mathrm{f}>\Delta \mathrm{f}_{\text {onset }}\right)$, the molecular network cannot be resolved in the topographic images (Fig. 3(a), left panel). As expected, the lateral resolution of the CPD images improves noticeably when reducing the tip-sample separation as shown by the comparison between the CPD images presented in Figs. 3(a) and 3(b) (right panels). In the later image, features with lateral dimensions of a few nanometers are clearly resolved. However, CPD modulations correlated with the molecular lattice appear only after a further decrease of the tip-surface

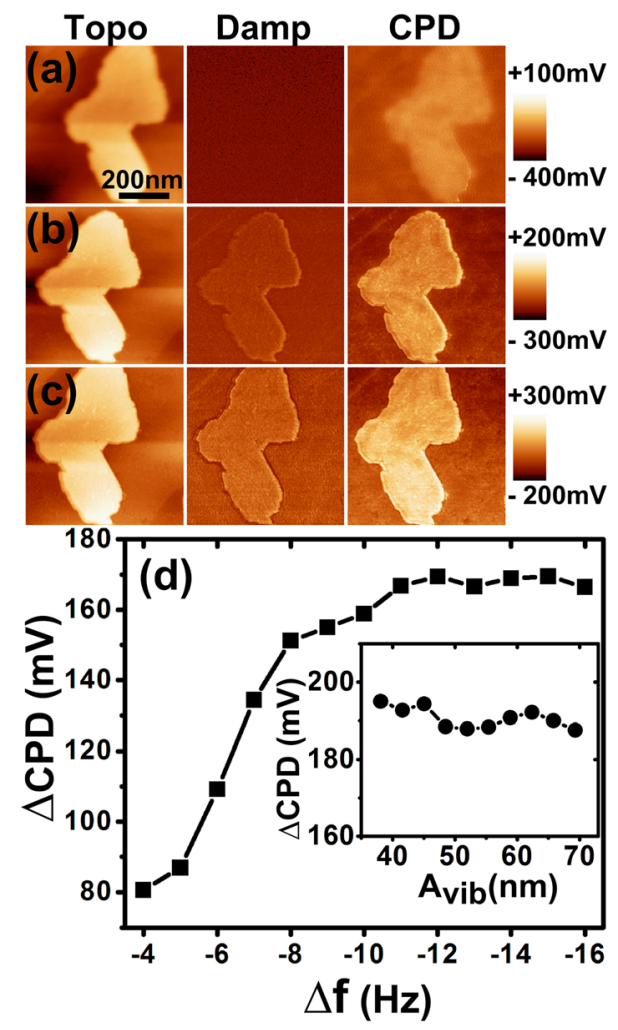

FIG. 2. (Color online) (a)-(c) Topographic (left panels), damping (middle panels), and CPD (right panels) images $(600 \mathrm{~nm} \times 600 \mathrm{~nm}, 512 \times 512$ pixels) of a FG1 stack on HOPG recorded with $\mathrm{A}_{\text {vib }}=55.7 \mathrm{~nm}$ and $\Delta \mathrm{f}=-4 \mathrm{~Hz}$ (a), $\Delta \mathrm{f}=-8 \mathrm{~Hz}$ (b), and $\Delta \mathrm{f}=-12 \mathrm{~Hz}$ (c). Color scales are given for the CPD levels only (right panels). (d) CPD difference between the stack and the substrate $\left(\triangle \mathrm{CPD}=\mathrm{CPD}_{\mathrm{FG} 1}-\mathrm{CPD}_{\mathrm{HOPG}}\right)$ as a function of the frequency shift, at constant vibration amplitude, $A_{\mathrm{vib}}=55.7 \mathrm{~nm}$. Inset: same parameter plotted as a function of the vibration amplitude, at constant normalized frequency shift $\gamma=\left(\Delta \mathrm{f} \times k \times \mathrm{A}_{\mathrm{vib}}{ }^{3 / 2}\right) / \mathrm{f}_{0}=-3.7 \mathrm{fN} \mathrm{m}^{1 / 2}$.

separation (Fig. 3(c), right panel), for set points corresponding to the saturation plateau in Fig. $2(\mathrm{~d})\left(\Delta \mathrm{f} \leq \Delta \mathrm{f}_{\text {sat }}\right)$.

Similar results have been obtained for QTF8 molecular stacks (Figs. 4(a)-4(c)), for which the topographic and dissipation images display identical contrasts than the ones reported in our previous works. ${ }^{16,19}$ Particularly, the dissipation (Fig. 4(b)) is higher over the wires with the $\mathrm{C}=\mathrm{O}$ bond pointing downward (the molecular electric dipole being oriented upward), consistently with Qplus AFM investigations ${ }^{19}$ carried out in the low amplitude regime.

The existence of well-defined non-equivalent molecular orientations was used to gain a deeper insight on the nature of the LCPD contrasts. First, the contrast difference between both kinds of molecular wires was estimated to be of $25 \pm 5 \mathrm{mV}$ from profile lines (not shown) extracted from the CPD images (Fig. 4(c)). Second, CPD(z) curves (Fig. 4(e)) were recorded at selected locations over the stack. Above all locations, the CPD shows a well marked drop from $\mathrm{z}=0$ (regulated set point) to $\mathrm{z} \approx 0.45$, and an almost constant value for greater tip-surface distances. The CPD difference between the curves at $\mathrm{z}=0$ is the same within the error bars than the one previously deduced from the CPD images. This confirms that most of the contrast should be explained by the distance dependence of the CPD. The point $\mathrm{z} \approx 0.45$ characterizes the onset of the short range forces, as shown by the damping curves given as an inset in Fig. 4(e). 


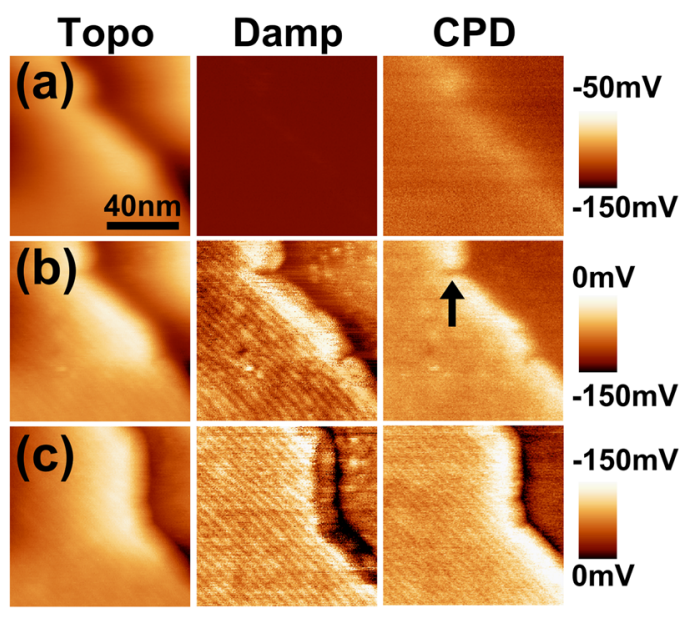

FIG. 3. (Color online) (a)-(c) Topographic (left panels), damping (middle panels), and CPD (right panels) high magnification images $(100 \mathrm{~nm}$ $\times 100 \mathrm{~nm}, 400 \times 400$ pixels) of the FG1 stack recorded with $\mathrm{A}_{\mathrm{vib}}=55 \mathrm{~nm}$ and $\Delta \mathrm{f}=-4 \mathrm{~Hz}$ (a), $\Delta \mathrm{f}=-6 \mathrm{~Hz}$ (b), and $\Delta \mathrm{f}=-11 \mathrm{~Hz}$ (c). At $-6 \mathrm{~Hz}$, a lateral resolution of a few nanometers is achieved in CPD images, as highlighted by the black arrow which pinpoints a defect at the edge of the molecular stack in (b) (right panel). CPD modulations correlated with the molecular lattice appear for $\Delta \mathrm{f} \leq \Delta \mathrm{f}_{\text {sat }}$, as shown by the data in (c).

Above that threshold position, the SRE forces do not contribute to the KPFM compensated CPD, which directly reflects the potential variations of the imaged surface. Reducing the tip surface separation, a crossover occurs between the LR and SR regimes, due to the increasing influence of SRE forces. At the smallest tip-surface separations, the "local" CPD becomes dominated by the contributions of SRE forces, accounting for the appearance of modulations correlated with the molecular lattice in CPD images.

However, similarly to the case of atomic contrasts, these variations cannot be directly attributed to local changes in the surface potential, due to non-conventional proximity effects between the tip apex and the surface. Further NCAFM/KPFM studies are planned to address the exact nature of the tip-surface interactions, such as quasi constant height CPD imaging and low temperature measurements.

In summary, SRE forces can contribute significantly to the LCPD contrasts in pi-conjugated stacks. Alternatively, our results demonstrate that the influence of the SRE forces can be minimized by working near the onset of the damping contrast. This provides an interesting hint to adjust the tipsurface separation for achieving an optimal lateral resolution in "conventional" CPD images (i.e., images registered at tipsurface separation out of the range of SRE forces) on organic layers.

SPM facilities used for the experimental work within UMR5819-SPrAM have been funded by the French "Recherche Technologique de Base" Program. M.L. thanks SERC (Swedish e-Science Research Center) for funding and SNIC for providing computer resources.

${ }^{1}$ H. Hoppe, T. Glatzel, M. Niggemann, A. Hinsch, M. Ch. Lux-Steiner, and N. S. Sariciftci, Nano Lett. 5, 269 (2005).

${ }^{2}$ E. J. Spadafora, R. Demadrille, B. Ratier, and B. Grévin, Nano Lett. 10, 3337 (2010).
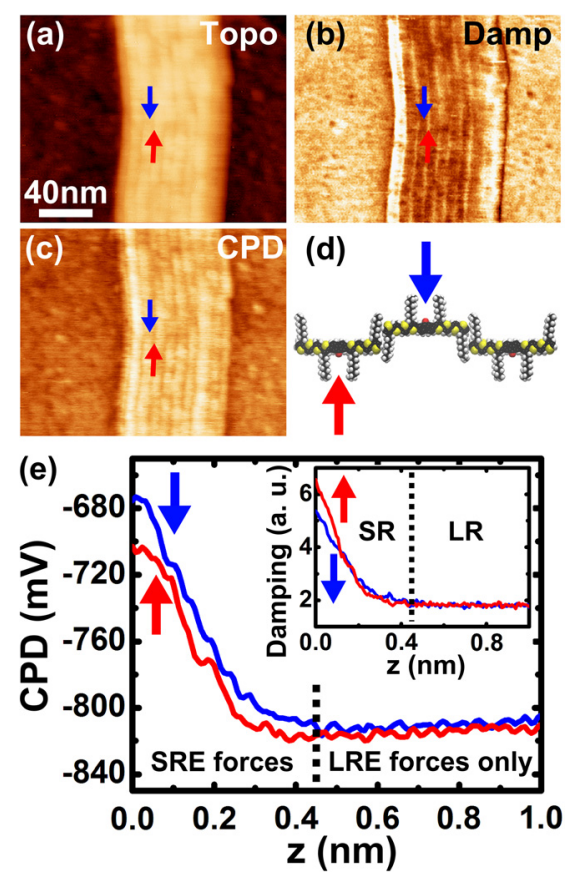

FIG. 4. (Color online) (a)-(c) Topographic (a), damping (b), and CPD (c) images $(200 \mathrm{~nm} \times 156 \mathrm{~nm}$, pitch $=0.33 \mathrm{~nm})$ of a QTF8 stack on HOPG recorded with $\mathrm{A}_{\mathrm{vib}}=35 \mathrm{~nm}$ and $\Delta \mathrm{f}=-20 \mathrm{~Hz}$. (d) Section (simulation from molecular mechanics) of an edge-on stack. Arrows pointing up and down highlight the locations corresponding to the two different molecular orientations. (e) CPD curves (averaged from several data) as a function of the tip-surface separation $\left(\Delta \mathrm{f}=-25 \mathrm{~Hz}, \mathrm{~A}_{\mathrm{vib}}=41 \mathrm{~nm}\right)$, recorded over the two different molecular orientations within a QTF8 stack, as highlighted by the arrows in (d). Inset: damping curves recorded simultaneously with the CPD data.

${ }^{3}$ L. Bürgi, H. Sirringhaus, and R. H. Friend, Appl. Phys. Lett. 82, 1482 (2003).

${ }^{4}$ T. Hallam, M. Lee, N. Zhao, I. Nandhakumar, M. Kemerink, M. Heeney, I. McCulloch, and H. Sirringhaus, Phys. Rev. Lett. 103, 256803 (2009).

${ }^{5}$ T. Shiota and K. Nakayama, Jpn. J. Appl. Phys. 41, L1178 (2002).

${ }^{6}$ T. Eguchi, Y. Fujikawa, K. Akiyama, T. An, M. Ono, T. Hashimoto, Y. Morikawa, K. Terakura, T. Sakurai, M. G. Lagally, and Y. Hasegawa, Phys. Rev. Lett. 93, 266102 (2004).

${ }^{7}$ F. Krok, K. Sajewicz, J. Konior, M. Goryl, P. Piatkowski, and M. Szymonski, Phys. Rev. B 77, 235427 (2008).

${ }^{8}$ G. H. Enevoldsen, T. Glatzel, M. C. Christensen, J. V. Lauritsen, and F. Besenbacher, Phys. Rev. Lett. 100, 236104 (2008).

${ }^{9}$ S. Sadewasser, P. Jelinek, C.-K. Fang, O. Custance, Y. Yamada, Y. Sugimoto, M. Abe, and S. Morita, Phys. Rev. Lett. 103, 266103 (2009).

${ }^{10}$ L. Nony, F. Bocquet, C. Loppacher, and T. Glatzel, Nanotechnology 20, 264014 (2009).

${ }^{11}$ M. P. Nikiforov, U. Zerweck, P. Milde, Ch. Loppacher, T.-H. Park, H. T. Uyeda, M. J. Therien, L. Eng, and D. Bonnell, Nano Lett. 8, 110 (2008).

${ }^{12}$ Th. Glatzel, L. Zimmerli, S. Koch, S. Kawai, and E. Meyer, Appl. Phys. Lett. 94, 063303 (2009).

${ }^{13}$ T. Ichii, T. Fukuma, T. Yoda, K. Kobayashi, K. Matsushige, and H. Yamada, J. Appl. Phys. 107, 024315 (2010).

${ }^{14}$ F. Lincker, N. Delbosc, S. Bailly, R. DeBettignies, M. Billon, A. Pron, and R. Demadrille, Adv. Funct. Mater. 18, 3444 (2008).

${ }^{15}$ F. Lincker, B. Heinrich, R. De Bettignies, P. Rannou, J. Pécaut, B. Grévin, A. Pron, B. Donnio, and R. Demadrille, J. Mater. Chem. 21, 5238 (2011).

${ }^{16}$ B. Grévin, R. Demadrille, M. Linares, R. Lazzaroni, and Ph. Leclère, Adv. Mater. 21, 4124 (2009).

${ }^{17}$ See supplementary material at http://dx.doi.org/10.1063/1.3662850 for NC-AFM images and detailed simulations (molecular mechanics and dynamics) of FG1 self-assembly on graphite.

${ }^{18}$ M. Józefowicz and J. R. Heldt, Spectrochim. Acta, Part A 67, 316 (2007).

${ }^{19}$ J. Hayton, F. Lincker, R. Demadrille, M. Linares, M. Brun, and B. Grévin, Appl. Phys. Express 2, 091501 (2009). 